\title{
Valoración de la competencia morfosintáctica en el niño de dos años y medio
}

\author{
GERARDO AGUADO \\ Centro de Orientación y Rehabilitación Psicopedagógica \\ Huarte de San Juan

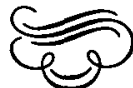 \\ Resumen
}

El objetivo del trabajo es doble: $1 .^{\circ}$ Investigar el desarrollo morfosintáctico de los niños de dos años y medio, considerando esta edad como clave a causa de la primera expansión sintáctica y saber si la longitud media de enunciado (LME) puede ser una buena medida de la complejidad morfosintáctica del hablante infantil. 2. ${ }^{\circ}$ Proporcionar un instrumento para la acción logopédica relacionada con los retrasos y trastornos en la adquisición gramatical.

Para ello se tomaron grabaciones de conversaciones semi-dirigidas de 24 niños de ambos sexos de dos años y medio. Se rastreó la presencia, en dichas conversaciones, de signos morfosintácticos que se agruparon bajo trece titulos.

Se halló la correlación existente entre la riqueza gramatical de cada sujeto (considerada como la suma de los signos morfosintácticos empleados, puntuando con 1 cada uno de los dichos signos) y su $L M E(\bar{X}=2,33, D S=0,78)$, balländose que ésta podía ser una buena medida de aquélla $(r=0,8402, p<0,01)$, sin tener en cuenta, por lo tanto, las transformaciones presentes en cada enunciado.

Finalmente, ordenando los sujetos de menor a mayor riqueza gramatical, se entresacan 10 pasos para facilitar la acción logopédica en el área morfosintáctica.

Palabras clave: Adquisición del lenguaje, competencia morfosintäctica, logopedia.

\section{Apprasial of the morpho-syntactic competence in a $2 \frac{1}{2}$ years old child}

\section{Abstract}

The aim of this work is double: 1) To study the development of morphosyntax in children aged 2; 6 considering this age to be the key age because of this first syntactic expansion and to find out whether the mean length of utterance (MLU) can be a good measure of morphosyntactic complexity in children. 2) To provide a tool for logopedagogical action in connection with delays and problems in grammatical acquisition.

To these purposes semi-directed conversations of 24 two-and-a half-year-olds of both sexes were recorded. The presence of morphosyntactic signs in said conversations was studied and grouped into 13 categories.

The correlation between each subject's grammatical richness (taken in terms of the total number of morphosyntactic signs used and counting each sign as 1) and this $M L U(\mathbb{X}=2,33$, $S D=0,78)$ was found. It was found that $M L U$ could be a good measure of grammatical richness $(r=0,8402, p>0,01)$ without considering, therefor, transformations underlying the surface structure of each utterance.

Finally, after ordering the subjects from least to maximum grammatical richness, ten steps to facilitate logopedagogical action in the are of morphosyntax were selected.

Key words: Appraisal, morphosyntactic competence, logopedagogical action.

Dirección de los autores: Centro de Orientación y Rehabilitación Psicopedagógica Huarte de San Juan. Avenida Conde Oliveto, 2, 3. Izda. 31002 Pamplona.

Original recibido: Mayo 1987. Revisión recibida: Junio 1988. Aceptado: Junio 1988. 


\section{INTRODUCCION}

El objeto del presente trabajo fue hacer una aproximación al análisis del desarrollo morfosintáctico del niño de 2 años y medio.

Detrás de este objetivo, y anterior a él, existía un interés más global y totalizador hacia el esclarecimiento de una secuencia de desarrollo morfosintáctico, general y aplicable a todos los niños creadores-aprendices del castellano.

Con respecto al primer punto, puede decirse que no existe un número extraordinariamente grande de trabajos como para hacer ociosa e inútil una pequeña aportación más. Más bien al contrario. En este sentido, seguimos manteniendo una dependencia, cada vez menor, afortunadamente, de estudios hechos en otras lenguas, sobre todo el inglés. A veces, en el trabajo de reeducación nos hemos visto obligados a entresacar explicaciones o secuencias de estudios morfosintácticos de otras lenguas, para aplicarlas, adaptándolas, no siempre con fortuna, a interrogantes planteados en el tratamiento logopédico de un niño concreto.

Ello no significa que nuestro panorama esté desierto de aportaciones muy valiosas sobre este tema. Basta recordar los trabajos de Hernández-Pina 1984, Gili Gaya 1974, Soler 1984, López Ornat 1984...

En relación al segundo objetivo y más práctico valgan las siguientes reflexiones.

Cuando se habla de logopedia a los padres de los niños afectados por algún trastorno de habla o de lenguaje, aquéllos suelen identificar el término de logopedia con «enseñar a pronunciar bien», para que se entienda mejor a su hijo. Normalmente, es necesaria una explicación adicional para dar luz al hecho de que el aspecto fonológico no es el único que se tiene en cuenta en el examen del lenguaje. Este hecho sería irrelevante si esta misma situación, llamémosla de vacío cultural, no se diera incluso en muchos profesionales de la psicología, incluso de la psicología escolar.

Esto ha hecho que muchos logopedas, formados a base de cursillos intensivos, de prácticas solamente, etc. (situación corregida presumiblemente por las EE.SS. de Logopedia) no hayan prestado atención al aspecto morfosintáctico como susceptible de alteración y, consiguientemente, de recuperación. Solamente se consideraba esta posibilidad en los casos de afasia. Sin embargo, es evidente que se producen retrasos, ciertos crecimientos disarmónicos de los diversos aspectos del lenguaje que pueden afectar a la morfosintaxis.

Desde este punto de vista, es muy importante tener una idea clara de la secuencia de desarrollo morfosintáctico. Porque ésta será la que posibilite al técnico en patología del lenguaje proponer modelos de frases ordenados, según dicha secuencia de crecimiento, «jugar» a cambiar el significado de la frase manipulando ciertas palabras, ciertos morfemas, etc, enriquecer, en fin, sus posibilidades y realizaciones cognitivas, juntamente con el enriquecimiento de sus formas gramaticales.

Este aprendizaje lo realiza el niño a partir de los ajustes que hace la madre de su lenguaje (Wyatt citada por Richelle, 1971). De este modo, el niño dispone de un modelo cercano, pero más complejo que el suyo. Esta función pedagógica del lenguaje del adulto es inconsciente por parte de este último. Y al ajuste de lo que el niño puede entender y expresar con el lenguaje del adulto es automático y se da incluso en las conversaciones entre un niño de 4 años y otro más pequeño (Gelman y Shatz citadas por Bruner, 1983). Esta función pedagógica sólo está en la mente del observador, ya que lo que se plantea entre 
madre e hijo es algo mucho más pragmático; en palabras de Bruner (1983): «Parecía no haber ninguna prueba de que la madre hubiera modelado deliberadamente las formas gramaticales que aparecieron en el habla del niño. Las formas gramaticales correctas, más bien se ofrecieron para quitarle ambigüedad a las interpretaciones de la petición del niño... Eran esfuerzos genuinos, no pedagógicos, que efectuaba la madre para entender el enunciado del niño. Está claro que estos enunciados servían como modelos, pero no eran, por así decir, ofrecidos a priori para ser imitados» (Bruner 1983).

No obstante, y considerando que la función del logopeda es esencial y conscientemente pedagógica, cabe suponer que impelidos por conseguir ciertas adquisiciones, fonemáticas, por ejemplo, no se tengan suficientemente en cuenta lo adquirido y por adquirir en la morfología y sintaxis.

Por ello y por otras razones derivadas del contacto directo, diario y largo en el tiempo, con logopedas y niños con alteraciones de lenguaje, creemos que no es bueno dejar el desarrollo morfosintáctico en ciertos niños con trastornos de lenguaje al mero automatismo. Es necesario un estudio pormenorizado del desarrollo de todas las formas morfológicas y sintácticas, que sirva para evaluar el momento evolutivo particular de un niño y que brinde las estrategias pertinentes y oportunas al logopeda (incluso, que le permita modificar el lenguaje que va a emplear con el niño), para favorecer dicho desarrollo.

El presente trabajo trata simplemente de dar luz a un pequeño fragmento de esa secuencia general.

\section{METODO}

\section{Sujetos}

Los sujetos fueron 24 niños de ambos sexos, 10 niñas y 14 niños, de dos años y medio \pm 7 días, que asisten a las guarderías municipales monolingües de Pamplona. El nivel socioeconómico familiar va desde el trabajador en paro hasta el universitario con libre ejercicio de su profesión.

El $55 \%$ de los niños son hijos únicos. El $45 \%$ tienen un hermano/a y el $5 \%$ tienen dos hermanos o hermanas. Del grupo que tiene un hermano, el $62,5 \%$ de nuestros sujetos van en segundo lugar. Los sujetos del tercer grupo van todos en último lugar.

La mitad de la muestra entró en el curso 85-86 (cuando se tomaron las grabaciones) en la guardería. La cuarta parte ingresó en guardería en el curso 84-85 y la otra cuarta parte lo hizo en el 83-84 (siendo lactantes).

\section{Material}

El empleado fue: un magnetofón, un cuento (Un caballo y un perro. Una cabra y un ganso. Escrito e ilustrado por Alice y Martin Provensen. Ed. Plaza y Janés), el material gráfico del NSST (Northwestern Syntax Screening Test) de Laura Lee y algunos juguetes: un camión volquete cargado de piezas de colores, una granja con 10 animales, vallado, tractor y remolque y granjero.

\section{Procedimiento}

Se tomaron muestras de lenguaje de media hora por cada niño. Las muestras se tomaron todas por la mañana, en el despacho del/de la responsable de la 
guardería en la que se encontrara el niño. Todas las muestras fueron tomadas por el autor del presente trabajo, encontrándose sólo con el niño, salvo en cuatro casos a causa de la inhibición manifestada: la conversación de uno de esos casos fue grabada por su educadora habitual, las de dos fueron grabadas por el autor acompañado de la educadora y una cuarta fue grabada por el autor, estando el niño acompañado de otro niño, amigo suyo.

La grabación transcurría de la siguiente forma: los 5 ó 10 primeros minutos se empleaban en jugar con los juguetes. Se iniciaba la grabación una vez que el niño comenzaba a emitir alguna expresión, y ya no se paraba de grabar, salvo que el niño tuviera que salir de la habitación. La mitad del tiempo restante ise empleaba en mirar y hablar sobre el cuento y la otra mitad en mirar y hablar sobre el NSST.

En la 1..$^{\mathrm{a}}$ parte (juguetes) se intentaba crear un ambiente agradable y distendido, que se consiguió en todos los casos, aún en los que hubo ayuda de terceras personas. Se recogieron gran cantidad de interjecciones, ruidos de animales y lenguaje espontáneo. La comunicación estaba condicionada por la actividad y las expresiones del niño.

En la 2.a parte, (cuento), el observador proponía al niño situaciones en las que se buscaba fundamentalmente la expresión de mòrfemas verbales, comprensión de comparativos, utilización de artículos, etc. Las ilustraciones del cuento, por otra parte, daban suficientes bases para la producción de abundante lenguaje espontáneo.

En la 3. parte (NSST), se buscaba la expresión de preposiciones, algunos morfemas verbales (el inglés es menos rico que el español a este respecto) y el lenguaje espontáneo era más reducido.

No obstante, los límites impuestos en principio a estas tres partes eran rotos con frecuencia por los niños (era algo que no sólo se esperaba, sino que era deseable) haciendo virar la conversación a otros temas de su vida personasl y/o familiar, retornando a los juguetes, al cuento, a otras láminas del NSST, etc. Pero, en cualquier caso, se proponían al niño estímulos para recabar toda la información sobre su riqueza gramatical.

Posteriormente, se procedió a la transcripción íntegra de todas las grabaciones.

Realizadas las transcripciones, se procedió a la observación de la presencia o ausencia de los elementos sintácticos y morfológicos más interesantes. Estos elementos pertenecen a distintos niveles de profundidad: desde elementos que contribuyen a la determinación de funciones semánticas primarias (interrogación, pasivación, roles de agente, paciente, etc.) y secundarias (mofemas verbales, género, número, etc.) hasta signos de superficie para combinación de proposiciones (Bronckart, 1977).

Se midió también la longitud media de enunciado.

\section{RESULTADOS Y COMENTARIOS}

Teniendo en cuenta lo que dijo más arriba sobre la relativa regularidad en la aparición de los signos gramaticales, se han tomado las observaciones realizadas sobre los 24 sujetos y, a pesar de tener todos dos años y medio, se han ordenado de menos a más riqueza gramatical, pretendiendo con ello dar una idea del orden de adquisición de los elementos morfosintácticos. Se podría suponer que la media de las puntuaciones obtenidas por los sujetos (entendiendo que 
se valora con 1 punto la presencia segura de dichos elementos) indicaría la riqueza gramatical del niño tipo de dos años y medio. Pero lo más importante respecto al objetivo de este trabajo es un orden que pueda ser utilizado en la actividad logopédica.

\section{Interrogación}

La pregunta, en esta edad, es el sistema habitual para solicitar información. Se hacen empleando frases afirmativas o negativas con la entonación apropia$\mathrm{da}$, e iniciándolas con pronombres y adverbios interrogativos.

Los resultados en nuestra muestra son:

Porcentaje de niños que hacen preguntas marcadas sólo con entonación: $100 \%$.

Porcentaje de niños que hacen preguntas con iniciador interrogativo: $79,17 \%$.

De las partículas interrogativas, la más empleada es sin duda el pronombre qué en preguntas del tipo: ¿qué es esto?

Es tan frecuente su uso que se emplea como comodín en frases interrogativas que exigirian otras partículas:

Sujeto 10: esto ¿qué se llama? ¿qué es éste?

Al sujeto 5 (S5) la expresión ¿qué? le sirve hasta para responder:

Observador: ¿qué es esto?

SS: ¿qué? un gato

En este sujeto dicha expresión tiene en muchas ocasiones una función fática. En su media hora de grabación se contabilizan 99 ¿qué?

No son infrecuentes, en los sujetos de poca riqueza gramatical, bastantes dificultades para la comprensión de las preguntas iniciadas con qué y quién antecedidas por una preposición. Por ejemplo: el citado S5 no entiende las preguntas: ¿de qué? y ¿de quién?

Las preguntas iniciadas con adverbios interrogativos (dónde, cómo, cuándo, etc.) corresponden a los sujetos de más riqueza gramatical, aunque su uso, incluso entonces, es acaso, solicitando la información de forma indirecta con preguntas tipo: $y$ ¿éste? o $y$ ¿esto?

No es en absoluto sorprendente que la interrogación sea el signo gramatical más establecido a esta edad, ya que supone un elemento imprescindible en la comunicación. Hernández-Pina ya encuentra este fenómeno suprasegmentario en la etapa del balbuceo (Hernández Pina, 1984).

\section{Negación}

Porcentaje de sujetos que niegan con el adverbio no: $100 \%$, de niños que niegan con oraciones negativas completas: $79,17 \%$ ).

También en este apartado la negación más evolucionada coincide con la mayor riqueza gramatical.

La utilización del adverbio no (y sí) es general. Sin embargo, el S1 (el de menor riqueza gramatical) no emplea en toda la grabación el adverbio afirmativo, ni ninguna expresión sustitutoria.

No obstante, existe cierta rigidez en el uso de frase negativa, y nuestros sujetos no echan mano del adverbio tampoco (sólo lo utilizan el 12,50 \%) que manifestaría un afianzamiento en negaciones sucesivas.

Al contrario, el adverbio también, cuya función corresponde a la del tampoco en el caso de la afirmación, es usado con mucha más profusión (el 75 \%). 
No resulta fácil explicar esta aparente contradicción, considerando que la negación explícita aparece antes que la afirmación en el desarrollo del lenguaje, si no es a partir del modelo propuesto por el adulto en sus conversaciones con los niños. Habría que suponer, entonces, que el adulto utiliza mucho más frecuentemente el tándem sí.. también que el no... tampoco. Pero no se disponen de datos que avalen o contradigan esta suposición.

Otro tanto cabe decir del empleo de la conjunción $y$ para manifestar afirmaciones sucesivas, y de la conjunción $n i$ para las negativas. El uso de $y$ para tal fin es más frecuente que el de $n i$, que sólo aparece una vez en el S16, en una emisión incompleta.

O: si hay letras

S16: no bay nada

O: si, mira

S16: ni un gato ni

en cambio emisiones tipo:

O: es muy presumida esta cabra

S19: sí, y ésta, mira

o esta otra conversación:

S23: que me ha limpiau estos zapatos

$\mathrm{O}:$ ite ha limpiado estos zapatos?

S23: $y$ la mano

$\mathrm{O}$ : oye, cuando te vas a la cama, entonces...

(es un intento de cambiar de tema)

S23: $y$ los dedos

O: $y$ los dedos. Cuando te vienes...

(otro intento de cambiar de tema)

S23: $y$ esto (se señala el brazo)

están presentes en muchos de nuestros sujetos.

A veces, para manifestar negaciones sucesivas emplean $y$ no:

$\mathrm{O}$ ¿ ¿qué hace el tractor?

S3: aquí y aquí no y aquí no (señala ruedas que no giran)

\section{Modelos oracionales}

El modelo más simple es el compuesto por el sujeto (S) y el verbo (V). El S1 es el único modelo que emite:

S1: se ha caído

La emite sólo una vez y es repetición de la dicha por el observador. El sujeto está elíptico, como buena parte de las oraciones producidas por los niños. Esta tendencia a eliminar el sujeto explícito es general en los hablantes castellanos. Los morfemas verbales relativos a la persona son tan claros y marcados que se hace innecesaria la explicitación del sujeto en bastantes casos.

Después, la estructura que aparece en todos los demás casos $(95,98 \%)$ es SVPr. (Sujeto-verbo-predicativo). El verbo suele ser el llamado típicamente copulativo (el verbo ser), aunque también se produce este modelo con el verbo llamarse.

Respecto al predicativo, se emplean adjetivos y participios pasados, sobre todo (los ejemplos de este modelo son muy numerosos).

El modelo $S V C d$ (sujeto-verbo-complemento directo) aparece en el 91,66 \% de los sujetos, lo mismo que $S V C a$ (sujeto-verbo-complemento adverbial).

Respecto al modelo $S V C d$ puede decirse que su construcción es muy simple, 
no necesitando de partícula conectiva alguna para enlazar los dos elementos de sintagma verbal (salvo cuando el $C d$ es una persona, en cuyo caso necesita la preposición $a$ ).

Sólo 4 de nuestros sujetos $(16,66 \%)$ utilizan una oración subordinada sustantiva como $C d$ (el S10 con la conjunción que elíptica y el S18 con toda la oración principal también elíptica):

S23: ha dicho Dosa Mari te no toma taamelos (= ha dicho Rosa Mari que no coma caramelos).

En el modelo $S V C a$, el $C a$ suele necesitar una preposición (la más frecuentemente utilizada es en, véase apartado de preposiciones, para marcar un complemento espacial).

Pero buena parte de la $\mathrm{Ca}$ de nuestros sujetos son adverbios (de lugar, de modo y de tiempo, sobre todo), que no necesitan de partículas de enlace con el verbo. Por tanto, el modelo $S V C a$, es tan simple como el modelo $S V C d$, al tratarse sólo de una «acumulación» de 3 palabras cargadas de significado.

Cuanto mayor es la tiqueza gramatical más preposiciones se emplean, y aparecen además oraciones subordinadas (temporales, de modo, etc.) como $\mathrm{Ca}$ :

S24: ... y cuando no es de noche pues van a levantarse los cerdos.

El modelo que más aparece tras los cuatro citados anteriormente, es, curiosamente, un modelo de 4 elementos: $S V C d C a(62,5 \%)$; aunque quizás no resulte tan curioso si tenemos en cuenta que este modelo puede ser el resultado de realizar dos comentarios ya sobradamente dominados, $C d$ y $C a$ ) de un mismo tema (S), utilizando el mismo núcleo en el comentario (V), es decir, el modelo que nos ocupa sería el resultado de la unión de los dos anteriores con un mismo $\mathrm{S}$ y un mismo $\mathrm{V}$.

El modelo SVCi (sujeto-verbo-complemento indirecto) es utilizado por el $29,16 \%$ de nuestros sujetos. Nótese la gran diferencia de niños que emplean este modelo respecto al anterior, y éste respecto a los 4 primeros.

Los siguientes modelos son:

ViCd (verbo impersonal-complemento directo): $25 \%$

ViCdCa: $25 \%$.

Los niños que utilizan estos modelos coinciden bastante exactamente con los niños de mayor riqueza gramatical, aunque la dispersión se va haciendo un poco mayor: El modelo $\mathrm{ViCd}$ es empleado por los sujetos 10, 11, 16, 21, 22 y 24; el modelo $\mathrm{ViCdCa}$, por los sujetos 10,16, 18, 19, 20 y 24 .

Respecto al hecho de la coincidencia de resultados para ambos modelos, es bastante lógica, considerando que el $V i$ más utilizado ha sido bay que exige, casi necesariamente un $C d$ y un $C a$ (un adverbio de lugar, normalmente).

El siguiente modelo es $S V C d C i$, usado por el $20,83 \%$ de los sujetos.

Los siguientes modelos son empleados tan solo por los sujetos de mayor riqueza gramatical, esto es, los sujetos numerados con el $17 \mathrm{y}$ posteriores, con alguna ligera dispersión hacia el sujeto 14 .

El orden, de mayor a menor porcentaje, es decir, de menor a mayor dificultad, es el siguiente:

ViCa: $12,5 \%$

SVCaCa: $12,5 \%$

SVCdCiCa: $12,5 \%$

SVCiCa: $8,33 \%$

SVCaCa: $8,33 \%$

SVPrCa: $4,16 \%$ 
Es preciso hacer alguna aclaración a propósito del verbo (V).

En muchas ocasiones el $\mathrm{V}$ no tiene una forma simple, bien sea por presentarse en un tiempo simple o por presentarse con el auxiliar haber en los tiempos compuestos. Nuestros sujetos utilizan, además de éstas, otras formas complejas, que indican otros valores aspectuales, pero que en la nomenclatura de los modelos oracionales aparecen representados por una sola $\mathrm{V}$.

Se hace referencia a las formas ir a + infinitivo (aspecto de futuro), querer + infinitivo (aspecto de la actitud de voluntad) estar + gerundio (aspecto continuativo), etc. En el presente trabajo son consideradas en conjunto como el núcleo del sintagma verbal, por lo que se han señalado con una sola $\mathrm{V}$.

Con respecto a otras formas rastreadas, ninguno de nuestros sujetos empleó la voz pasiva, ni tampoco la comprendió. Se cuidó de que en la imagen que se les presentaba cualquiera de los dos sustantivos de la oración pudieran adoptar indistintamente el papel de agente y paciente. Concretamente era una lámina del NSST en la que un niño empuja un carro con una niña («la niña es empujada por el niño») y viceversa («el niño es empujado por la niña»). Esto es lógico, ya que si se les hubiera presentado estímulos como: «El cristal es destrozado por el niño», su inversa («el niño es destrozado por el cristal») carecería de sentido para el niño, lo que hubiera impedido a éste responder basándose en la gramática; su respuesta hubiera estado basada en aspectos semánticos, pertenecientes a la realidad vivida por el sujeto, despreciando los signos gramaticales: orden, preposición y forma verbal.

Otro tanto cabe decir de las oraciones comparativas. Se utilizó una sola forma:.. más... que y todos los niños respondieron invariablemente de la misma forma.

En una página del cuento empleado como material aparecen un caballo y unos pollos, y la situación se desarrollaba asi:

$\mathrm{O}$ : El caballo es más grande que el pollo ¿verdad?

S: $s \bar{i}$

O: Oye, y ¿el pollo es más grande que el caballo?

S: $s i$

\section{Coordinación}

El 41,66\% de nuestros sujetos utiliza de forma explícita oraciones compuestas coordinadas (Tabla I).

Estas oraciones han tenido por nexo la conjunción copulativa y y la adversativa pero.

La presencia de la conjunción copulativa es más extensa $(79,16 \%)$ que la de oraciones coordinadas. Esta aparente contradicción es fácilmente explicable.

Buena parte de estas conjunciones actúan de nexos demarcadores dentro de un sintagma nominal heterogéneo. Hay muchos ejemplos de ello. Basta uno como muestra:

S24: Hay cuatro... cuatro gallos... y unas gallinas.

Por tanto, estas conjunciones copulativas no unen dos oraciones sino que extienden el sintagma nominal, lo complejizan, como lo hacen la conjunción $o$ en bastantes casos, la preposición de (la mesa de madera es...») y el relativo que en una oración subordinada adjetiva.

El que las oraciones coordinadas sean menos frecuentes que las subordinadas se explica por el hecho de que aquéllas, por su propia condición de coordina- 
das, funcionan como principales que no precisan necesariamente el nexo (sobre todo, la conjunción copulativa) y, simplemente, se yuxtaponen.

Por otra parte, el propio sistema conversacional empleado, hace que las emisiones sean cortas, y que no se den muchas narraciones, en las que se tendería a emplear, presumiblemente más nexos coordinativos.

\section{Subordinación}

El $58,33 \%$ de nuestros sujetos emplean oraciones subordinales en los términos definidos en la tabla $I$.

\section{TABLA I}

Indices de coordinaciōn y subordinación, con las longitudes medias de enunciado (L.M.E.).

\begin{tabular}{|c|c|c|c|}
\hline SUJETOS & $\begin{array}{c}\text { INDICE } \\
\text { COORDINADAS }\end{array}$ & $\begin{array}{c}\text { INDICE } \\
\text { SUBORDINADAS }\end{array}$ & L.M.E. \\
\hline 1 & & & 1,06 \\
2 & & & 1,63 \\
2 & & & 1,70 \\
4 & & & 1,98 \\
5 & & & 1,43 \\
6 & & & 1,73 \\
7 & & & 1,64 \\
8 & & 1,45 & 2,05 \\
9 & & 0,74 & 1,82 \\
10 & 1,36 & 0,68 & 2,46 \\
11 & & & 1,92 \\
12 & & & 2,62 \\
13 & & 2,22 & 2,13 \\
14 & & 1,97 & 2,18 \\
15 & 0,49 & 2,60 & 2,55 \\
16 & 1,56 & 5,11 & 2,62 \\
17 & 3,65 & 10,13 & 2,93 \\
18 & 1,27 & 1,22 & 2,46 \\
19 & 0,61 & 4,32 & 2,74 \\
20 & 3,60 & 3,00 & 2,92 \\
21 & 1,00 & 5,60 & 2,97 \\
22 & 4,00 & 1,44 & 2,59 \\
23 & & 19,83 & 2,72 \\
24 & 6,33 & & 5,14 \\
\hline
\end{tabular}

Los índices de coordinación y subordinación se han hallado mediante estas fórmulas:

indice de coordinación $=\frac{\mathrm{n}^{\circ} \text { de oraciones coordinadas } \times 100}{\mathrm{n} .^{\circ} \text { total de oraciones }}$

indice de subordinación $=\frac{n^{0} \text { de oraciones subordinadas } \times 100}{n \cdot{ }^{0} \text { total de oraciones }}$

Se toman en cuenta en el n. ${ }^{\circ}$ de oraciones coordinadas y subordinadas sólo las que manifiestan en un solo enunciado las dos (o más) oraciones unidas con el nexo coordinativo, y la oración principal y subordinada con el nexo subordinativo, respectivamente.

Las oraciones de relativo (subordinadas adjetivas) son consideradas como subordinadas.

$\mathrm{Se}$ añaden las L.M.E., que se comentarán en el apartado 14 (longitud media de enunciado y su correlación con la riqueza gramatical). 
Es indudable que muchas yuxtaposiciones podrían ser interpretadas como oraciones subordinadas, pero se consideró que sólo era segura la existencia de una subordinación cuando aparecían todos sus elementos. De otra forma, podríamos caer en generalizaciones gratuitas.

La más común de las oraciones subordinadas es la de relativo: el $71,43 \%$ de los sujetos que emplearon subordinadas, incluían entre ellas oraciones de relativo. En el S20, por ejemplo, todas las subordinadas son de relativo.

El $35,71 \%$ de los sujetos que produjeron oraciones subordinadas, emplearon adverbiales comparativas. Normalmente estas operaciones eran elípticas, fuertemente elípticas, podríamos decir: faltaba la opración principal entera y el verbo de la subordinada, generalmente. Se podría pensar que parecía que eran ampliaciones de un sintagma nominal. Ejemplo:

O: ¿de qué color son?

S23: tomo $(=$ como) estos zapatos.

Un ejemplo en el que no falta la oración principal:

S20: tiene bigotes como tú

El S22 produce en una sola ocasión este tipo de subordinación, sin la oración principal, pero con la adverbial entera:

S22: como el que tienes tú en casa.

Pero emisiones como estas últimas son muy escasas. Las más frecuentes son las del tipo emitido por el S23.

También un $35,71 \%$ produce adverbiales de lugar. Y cabe decir lo mismo, respecto a cómo se producen, que lo que se ha dicho más arriba de las comparativas.

O: ¿por qué no se pinchará en los pies?

S23: none (= donde) esa bieba (= hierba)

$\mathrm{El}$ por qué se producen estas «fuertes» elipsis obedece a que el verbo y otros elementos de la oración subordinada son los mismos que los de la oración principal, por lo que es ocioso repetirlos. El lenguaje del adulto es también de este tipo, en lo que se refiere a las dos formas de subordinación comentadas.

Después vienen las oraciones subordinadas sustantivas (en función de $\dot{C} d$. Las adverbiales temporales, causales, condicionales y modales, por este orden.

Respecto a las oraciones introducidas por la conjunción porque, las menos están contabilizadas como oraciones subordinadas, ya que dicha conjunción, en muchos casos, sirve como comodín y/o como una forma de responder, que se antepone a una oración principal; ejemplo:

O: ¿qué tal te lo pasabas?

S17: porque sí

O: Sí, pero ¿cuándo ibas a la playa tü?

S17: porque iba a la playa

$\mathrm{O}:$ pero ¿cuándo?

S17: porque be ido a la playa a bañarme.

Para mayor información, se remite al lector al apartado conjunciones.

Todas estas formas de subordinación se encuentran sólo en las grabaciones de los niños de mayor riqueza gramatical (sujetos 18, 19, 20, 21, 22, 23 y 24).

\section{Género y número}

Todos nuestros niños dominaban estos elementos sintácticos. Sin embargo, el S1 no fue capaz de responder con palabras en plural a preguntas dirigidas 
con este fin, aunque pareció que hacía algún plural espontáneamente, difícil de captar, debido a dificultades de origen fonológico.

Este fue algo que hubo que tener en cuenta a la hora de consignar los morfemas de plural en muchos sujetos. Pero tanto al añadir el morfema -s, como el más complejo -es, nuestros sujetos manifestaron un buen dominio.

No obstante, se produjeron algunas maneras de formar el plural, no «ortodoxas», pero originales, en niños que, en otros momentos de la grabación, lo hacían según nuestros cánones gramaticales:

Teniendo presente una lámina del NSST, en la que hay cuatro dibujos, de los que dos representan un ciervo y dos ciervos se desarrolló el siguiente diálogo:

$\mathrm{O}:$ Aquí hay un ciervo ¿verdad? ¿y aqui?

S6: $n$ fo $m a \overline{~(=~ c i e r v o ~ o ~ c i e r v o s ~ m a ́ s) ~}$

Con respecto al género no hubo nada especial digno de notarse. Cuando se empleaban artículos u otro tipo de presentadores, las concordancias se producían de una forma aceptable.

\section{Artículos}

El $87,5 \%$ de los niños utilizaban bien los artículos. Predominaron las formas singulares. La diferente actualización que se hace del nombre según el artículo que se emplee (indeterminado y determinado) fue ejecutada generalmente de forma correcta.

El S1 (que supone el 4,16\%) no empleó artículos.

El $8,33 \%$ (los sujetos 3 y 6) usó una forma vocálica (una /a/ constreñida) como artículo para cualquier género y número, que aquí se denomina «protoartículo».

En el caso del S3, probablemente se debiera esto a una dificultad en el área fonológica, consecuente a una disfemia que le hacía deformar muchas de sus emisiones.

\section{Adjetivos presentadores}

En nuestra muestra aparecieron 4 clases de presentadores que corresponden a otras tantas formas de actualizar el nombre.

a) Adjetivos presentadores demostrativos.

El más utilizado con diferencia, fue este,-a (un $62,5 \%$ de los niños), en singular, en la mayor parte de los sujetos.

Ese-a fue producido por el $33,33 \%$ de los niños.

Aquel,-lla no apareció en ningún caso.

La distinción entre la forma presentadora y pronominal de estas palabras (esteéste, ese-ése, etc.) se realiza teniendo en cuenta si van acompañadas de nombre o no. Esto es fácil y simple.

Pero no resulta tan simple si se va un poco más allá de una sencilla regla gramatical.

Sólo en el caso de los demostrativos la forma pronominal es claramente más frecuente que la forma adjetiva. No ocurre así con los posesivos e indefinidos, como veremos más adelante. También son los demostrativos (sean adjetivos o pronombres) los que son susceptibles de ser acompañados por un gesto claro que evidencia nítidamente el objeto cuyo nombre se actualiza.

¿Qué significa todo esto? Muchos pronombres demostrativos podrían ser catalogados como adjetivos, ya que van acompañados por un gesto indicador que 
podría ser tomado (el gesto y el objeto señalado) como una «forma motora y deíctica» de sustantivo. Al menos, en bastantes casos, no es fácil asignar las palabras este-a y ese,-a a una clase definida de palabras que se denomine adjetivos o pronombres.

b) Adjetivos posesivos

El 41,66 \% utilizaron esta clase de palabras. La forma más frecuente fue la $1 .^{2}$ persona singular, después, y por este orden, el singular de la $2 .^{a}$ y $3 .^{a}$ persona. Las tres personas del plural no aparecieron en ningún caso.

Las formas posesivas que funcionan como atributo (mio, tuyo, etc) se observaron en el $37,5 \%$ de los casos. Predominaba el singular de la $1 .^{2}$ persona del singular, pero se dieron con relativa frecuencia (en el $44,4 \%$ de los sujetos pertenecientes a este grupo) formas plurales de la $1 .^{a}, 2 .^{a}$ y $3 .^{a}$ persona del singular. No se encontraron estos posesivos en ninguna de las tres personas del plural.

c) Adjetivos indefinidos.

Porcentajes de niños que usaron:

otro: $79,16 \%$

mucho: $20,83 \%$

poco: $8,33 \%$

todo: $8,33 \%$

alguno: $8,33 \%$

ninguno: $4,16 \%$

Predominaron las formas en singular. Por ejemplo, otro se usó siempre en singular (masculino y femenino), lo mismo que poco. Aparecieron dos plurales en mucbo. Todo fue empleado sólo en plural por los dos sujetos que lo usaron, lo mismo que alguno.

El adjetivo todos se empleó acompañada del artículo (todos los) y poco, con la preposicion partitiva de y del artículo indeterminado (un poco de).

d) Los adjetivos numerales fueron muy poco frecuentes. Por otra parte, su distribución no respondían a la mayor o menor riqueza gramatical, dependía más bien del conocimiento que tuvieran los niños de la cantidad.

\section{Pronombres}

a) Los pronombres personales, con función de núcleo (sujeto) fueron empleados por el $66,66 \%$ de los niños.

La forma más utilizada fue la $1^{2}{ }^{2}$ persona del singular (el $87,5 \%$ de los sujetos que produjeron este tipo de pronombres). El $50 \%$ usó la $2 .^{a}$ persona del singular y solamente el $12,5 \%$, la $3 .^{2}$ persona.

No se detectó ninguna forma en plural.

Los pronombres personales, con función de complemento fueron empleados por el 79,16\% de los niños. Las formas más frecuentes fueron las de $1 .^{\mathrm{a}}$ persona (sobre todo, me). Parecida frecuencia presentaron las formas de $3 .^{a}$ persona del singular le y lo, ésta última en posición enclítica, generalmente.

Las formas de la 2.a persona del singular aparecieron con bastante menos frecuencia.

Tampoco se encontraron producciones de las formas plurales de estos pronombres.

La diferencia, a favor de los personales complemento, entre los dos tipos de pronombres, se debe a que en español el uso de los primeros es optativo, pue- 
den ser eliminados, sin que existan, por ello, dificultades de comprensión. Es de todos bien sabido que en otras lenguas, su uso es imprescindible.

b) De los pronombres demostrativos, el más utilizado fue éste,a: el 91,66\% de los niños lo emplearon, casi siempre en su forma singular. De entre ellos, sólo el $22,72 \%$ produjeron la forma plural.

La forma neutra (esto) se detectó con bastante frecuencia: en el $75 \%$ de los casos. Esta forma es ambigua y sirve a la deixis como comodín. Siempre necesita de un gesto preciso, que elimine la ambigüedad. Su uso, dentro de cada caso, es bastante profuso. Con ello, se hace referencia también a lo dicho a propósito de los demostrativos en el apartado anterior.

En el $70,83 \%$ de los niños se encontró la forma ése,-am en singular. En el $17,64 \%$ de dichos niños se observó la presencia de las correspondientes formas en plural.

La forma neutra (eso) apareció en el $50,33 \%$ de nuestros sujetos.

No se encontró el pronombre demostrativo aquél en ninguna de sus formas.

c) Pronombres indefinidos

Porcentajes de niños en los que se encontraron:

otro: $62,5 \%$

mucho: $25 \%$

todo: $20,83 \%$

poco: $12,5 \%$

Se usaron, sobre todo en plural.

\section{Verbos}

Las formas menos significativas, desde el punto de vista del progreso gramatical, son el pretérito indefinido, que fue usado por los sujetos $5,13,19,23$ y 24 , el imperfecto de indicativo, que lo fue por los sujetos $14,15,16,18$ y 24, y el potencial simple que sólo lo utilizó el S15. Los demás tiempos se fueron encontrando, según su frecuencia, en los sujetos que manifestaron mayor riqueza gramatical (Ver Tabla II).

No obstante, aún incluso de las tres formas citadas, se percibe una ligera, pero clara tendencia al agrupamiento en los niños que emitieron enunciados más complejos.

El tiempo que más inestabilidad presentó, entendiendo por inestabilidad la frecuencia de usos «erróneos», fue el presente de subjuntivo. Véase este ejemiplo:

O: ¿Dónde lo tienes? Cómetelo (un caramelo)

S11: e vaya yo a la calle y me lo como

$\mathrm{O}$ : ¿cuándo te comerás el caramelo?

S11: antes, y vaya yo allá, entonces me lo como

En algún caso, aparece en oración principal con valor y función de imperativo:

S10: no pise (-pises) e agua ¿eh?

(Esta forma, el imperativo, no está contabilizada, por ser, el imperativo propiamente dicho, la raíz pura del verbo. Su presencia se observó en todos los sujetos que emplearon verbos. Además, entender el imperativo como modo verbal es un punto discutible. «Pensamos que el imperativo es un modo del habla y no de la lengua; pertenece únicamente al plano de la llamada al interlocutor, está fuera del sistema, como señalan E. Alarcos Llorach, G. Guillaume, M. Molho y otros» (Lamíquiz, 1975). Este autor, Lamíquiz, sólo considera dos modos (indicativos o tiempo in esse y subjuntivo o tiempo in fier) y un no modo (infinitivo o tiempo in posse). 
TABLA II

Uso de formas verbales. Porcentajes de Ss que emplean cada una de las formas

\begin{tabular}{|c|c|c|c|c|c|c|c|c|c|}
\hline Sujeto & Pret. Ind. & Pret. Perf. & Gerun. & Ir $a+\operatorname{lnf}$. & Imperf. Ind. & Press. Subj. & Pret. Ind. & Fut. Sim. & Pot. Sim. \\
\hline $\begin{array}{r}1 \\
2 \\
3 \\
4 \\
5 \\
6 \\
7 \\
8 \\
9 \\
10 \\
11 \\
12 \\
13 \\
14 \\
15 \\
16 \\
17 \\
18 \\
19 \\
20 \\
21 \\
22 \\
23 \\
24\end{array}$ & $\begin{array}{l}x \\
x \\
x \\
x \\
x \\
x \\
x \\
x \\
x \\
x \\
x \\
x \\
x \\
x \\
x \\
x \\
x \\
x \\
x \\
x \\
x \\
x \\
x\end{array}$ & $\begin{array}{l}\mathbf{x} \\
\mathbf{x} \\
\mathbf{x} \\
\mathbf{x} \\
\mathbf{x} \\
\mathbf{x} \\
\mathbf{x} \\
\mathbf{x} \\
\mathbf{x} \\
\mathbf{x} \\
\mathbf{x} \\
\mathbf{x} \\
\mathbf{x} \\
\mathbf{x} \\
\mathbf{x} \\
\mathbf{x} \\
\mathbf{x} \\
\mathbf{x} \\
\mathbf{x} \\
\mathbf{x}\end{array}$ & $\begin{array}{l}x \\
x \\
x \\
x \\
\\
x \\
x \\
x \\
x \\
x \\
x \\
x \\
x \\
x \\
x \\
x \\
x \\
x\end{array}$ & $\begin{array}{l}\mathbf{x} \\
\mathbf{x} \\
\mathbf{x} \\
\mathrm{x} \\
\mathrm{x} \\
\mathrm{x}\end{array}$ & $\begin{array}{l}x \\
x \\
x \\
x\end{array}$ & $\begin{array}{l}x \\
x \\
x \\
x \\
x\end{array}$ & $\begin{array}{l}\mathbf{x} \\
\mathbf{x} \\
\mathbf{x} \\
\mathbf{x}\end{array}$ & $\begin{array}{l}\mathbf{x} \\
\mathbf{x} \\
\mathbf{x} \\
\mathbf{x}\end{array}$ & x \\
\hline $\begin{array}{l}\text { Porcen- } \\
\text { tajes }\end{array}$ & 95,83 & 83,33 & 70,83 & 45,83 & 20,83 & 20,83 & 20,83 & 16,66 & 4,16 \\
\hline
\end{tabular}

Los signos $\mathrm{x}$ indican la presencia segura, de la que puede deducirse que el niño utiliza apropiadamente la forma de que se trata, de cada tiempo y modo verbal en cada uno de los sujetos.

Por otra parte, se dieron invitaciones a la acción no sólo por medio de imperativos, sino por medio de formas más complejas y evolucionadas:

S24: Pues a ver si... vamos

(En los puntos suspensivos produce una oración subordinada adverbial temporal con acuandos)

También, respecto al imperfecto del indicativo se producen «incorrecciones»:

O: pero ¿qué harias con la chaqueta?

S22: me la ponía

$\mathrm{O}$ : ¿y si lloviera?

S18: mañana babia tormenta

Las concordancias de personas con sus morfemas verbales, normalmente, eran ejecutadas correctamente, aunque se dieron faltas en dicha concordancia:

$\mathrm{O}$ : ¿sin manta?

S23: pero ahora bazo frio

Respecto a la comprensión de los tiempos verbales, se detectaron fallos en 
niños que en otros momentos de la grabación manifestaban un buen conocimiento de aquéllos:

O: Oye ¿cuándo te pondrás el abrigo?

S23: el año pasau.

Esto sucedía cuando los tiempos utilizados en la pregunta eran los menos frecuentes en las emisiones de los niños (futuro de indicativo, imperfecto de indicativo, etc.)

Formas verbales que expresan un significado más complejo y preciso fueron utilizadas por muy pocos sujetos. El S24 fue el que más precisión aspectual manifestó:

S24: por aquí suele estar una gineta

Por otra parte, creaba verbos nuevos a partir de un verbo conocido y de una partícula inseparable:

S24: una vaca agarrándose al coche ;Ay! que se desarrima.

La reflexiva es una voz que utilizaron prácticamente todos los niños (el $95,83 \%)$.

\section{Adverbios}

Los adverbios más utilizados, lógicamente, fueron: sí: 95,83 \% y no: $100 \%$.

Respecto a otros adverbios de afirmación y negación (también y tampoco, respectivamente, que M. Secollama «de relación con lo dicho: acumulación»), se remite al lector a lo dicho en el apartado 2, y es mostrativo de ellos la frecuencia con que se producen: también: $75 \%$ y tampoco: $12,5 \%$.

En relación a los adverbios que denotan circunstancias, los más frecuentes son, sin duda, los de lugar: ahĩ: 83,33\%; aquĩ: 79,16\%; allí,-ä; 62,5\%; debajo (abajo): 45,83 \%; arriba: 41,66 \%; encima: 29,16\%; detrás: 20,83 \%; lejos: $20,83 \%$; adentro: $20,83 \%$ y afuera: $16,66 \%$.

$A$ veces estos adverbios van unidos a preposiciones, o adjetivos formando una locución:

S6: p'allá, p'allá (= para allá).

S12: s'ba bajau p'aíba (= para arriba)

S15: abi alto.

Después de estos adverbios, los que con más profusión se presentaron fueron los de tiempo: abora: 41,66 \%; ya: $25 \%$; entonces: $25 \%$; mañana: $16,66 \%$ y luego: $16,66 \%$. Y siguen, con una frecuencia que va de 8,33 al 4,16\% otros adverbios de tiempo: siempre, hoy, ayer, pronto, de noche, tarde, aún y antes.

De los adverbios de modo, el más usado fue: así: $58,33 \%$, seguido de muy lejos por: bien: 16,66 \%; mal: 4,16\% y algunas locuciones (de mentiras, etc.) empleadas una sola vez.

Los adverbios de intensidad que se encontraron fueron: muy (mucho): $45,83 \%$, nada: $37,5 \%$ y más: $37,5 \%$.

Otro capítulo aparte lo forman los adverbios relativos, que hacen que toda una oración se «degrade» y se haga complemento, exigiendo para ello, en bastantes casos, formas verbales del modo subjuntivo. El porcentaje de niños que los emplearon es como sigue: donde (adverbio relativo de lugar): $20,83 \%$ y cuando (adverbio relativo de tiempo): 16,66 \%. No se encontraron adverbios relativos de intensidad (cuanto, etc.), ni de modo (como). Su presencia se dio en los sujetos de mayor complejidad gramatical en sus emisiones. En realidad, estos adverbios fueron encontrados en más sujetos que los señalados, sobre to- 
do cuando. Sin embargo, sólo se contabilizaron los que aparecían de forma inequívocamente espontánea, y no los que eran respuestas-eco a preguntas iniciadas con el interrogativo cuándo.

Los adverbios interrogativos aparecieron bastantes menos veces. Entre ellos, también se produjo el adverbio interrogativo de modo (cómo), aunque en un solo caso (S24).

\section{Preposiciones}

Los porcentajes fueron los siguientes: en: $79,16 \%$; con: $58,33 \%$; a (dirección): $58,33 \% ; a$ (con complemento indirecto o directo): $50 \%$; de (en locución, fundamentalmente con adverbios de lugar: encima de, etc. o como nexo de verbo y suplemento: tirar de, etc.): $50 \%$; de (materia): $33,33 \%$; de (pertenencia): $29,16 \%$; por ( = a través de): $29,16 \%$; de (procedencia): $12,5 \%$; sin: $12,5 \%$ y hasta (límite espacial): $4,16 \%$.

Se dieron emisiones de preposiciones muy primitivas, utilizando una o dos como comodines, en sustitución de todas las demás:

$\mathrm{O}:$ ¿dónde está el gato?

S6: a casa

En algunas ocasiones, el poco desarrollo fonológico hacía difícil la discriminación de la presencia o no de algunas preposiciones. La más afectada fueron a y de:

S12: el coche le pega e ten (= el coche le pega al tren).

No faltaron formaciones originales, utilizando los nexos preposicionales:

O: mira ¿qué es esto?

S15: un reloj de la hora

$\mathrm{O}:$ ¿dónde está este gato?

S19: aquí debajo

$\mathrm{O}:$ ¿debajo? ¿de dónde debajo?

S19: por el caballo (= debajo del caballo?)

que, por otra parte indican inestabilidad en su uso.

\section{Conjunciones}

Porcentajes: $y$ : 79,16\%; pero: 29,16\%; como (comparativa): 20,83\%; que (nexo para oración sustantiva): $16,66 \%$; porque: $12,5 \%$; si: $8,33 \%$ y para qué: $8,33 \%$

Respecto a la conjunción coordinativa $y$, no siempre cumplía esta función, sino que bastantes veces era el nexo demarcador en sintagmas nominales heterogéneros (ver apartado 4). No obstante, su posibilidad de ambigüedad ofrecía la posibilidad de servir de nexo en gran número de oraciones compuestas, incluso subordinadas.

Hubo un uso bastante profuso de la conjunción que con función expletiva:

S23: que le muéde el tonejo (que le muerde el conejo).

Incluso también apareció con esta función la conjunción pero.

La conjunción que más inestabilidad presentó en sus uso fue porque. Ya se ha apuntado algún ejemplo antes (apartado 5), pero hay bastantes más:

$\mathrm{O}:$ ite pasa algo malo cuando comes caramelos?

S23: porque me da Mary Carmen

$\mathrm{O}$ : ¿cómo que no sabes?

S3: porque no 


\section{O: lo ata de la cuerda ¿a dónde?}

S22: porque está saltando (el caballo del cuento)

O: ¿por qué está saltando?

S22: porque si. Mira.

$\mathrm{O}:$ ¿está saltando? (tono de duda)

S22: porque tiene la pata rota.

S19: sí. Los pollitos tienen pies porque no tienen.

14. Longitud media de enunciado (L.M.E.) y su correlación con la riqueza gramatical.

La L.M.E. se halló dividiendo el número total de palabras entre el número de emisiones, entre las que no se tuvieron en cuenta las emisiones ininteligibles y las incompletas, pero manteniendo las repeticiones espontáneas, las expresiones no oracionales y las respuestas anómalas.

Para ello, se definió «palabra» como lo que se halla entre dos espacios vacíos.

Como expresiones no oracionales se entendieron las llamadas verbales de atención (1), las imitaciones de ruidos de animales, salvo si estaban nominalizadas por medio del artículo, etc.

Como respuestas anómalas, se entendieron aquellas expresiones emitidas fuera del contexto de la pregunta, del tema de conversación y del material que se tuviera entre manos.

Ver cuadro I apartado 4.

La duda de si la L.M.E. puede ser un buen indicador de la complejidad gramatical (véase, por ejemplo, Crystal et al, 1981), nos llevó calcular el coeficiente de correlación entre L.M.E. y el número de elementos morfosintácticos (ya descritos en los apartados anteriores) hallados en las emisiones de los sujetos. Este coeficiente fue de $r$ : $\mathbf{0 , 8 4 0 2}$.

Este resultado es elocuente por sí mismo y necesita pocos comentarios. La alta correlación hallada apunta a lo expuesto por Lenneberg (1975) y Cazden y Brown (1975) sobre que la complejidad gramatical no estaría definida sólo por el número de transformaciones exigidas en las oraciones, sino, sobre todo, por la acumulación de especificaciones que exige la reducción del campo semántico de una palabra (Lenneberg) y por un «sentido acumulativo» de palabras (Cazden y Brown, 1975).

De todos modos, este coeficiente de correlación muestra que la L.M.E. por sí sola, es capaz de darnos una correcta información de la complejidad gramatical de un hablante infantil.

Datos relativos a la L.M.E. y a la riqueza gramatical:

L.M.E. $\bar{X}=2,33$ palabras y $\sigma=0,78$

Riqueza gramatical: se concede un punto por la presencia de cada uno de los elementos morfosintácticos estudiados. Se omiten puntuaciones derivadas de la estructura de frase u de los adverbios. Respecto a la estructura de frase, resultó muy difícil tener en cuenta el número de frases emitidas y su complejidad a la vez, con el fin de asignarles puntuaciones.

Respecto a los adverbios, si se le hubiera asignado un punto a la presencia de cada adverbio, hubiera resultado un enorme peso específico de estas palabras, respecto de otras formas, por ejemplo los morfemas verbales, en el campo cuantitativo de riqueza gramatical.

No obstante, cuando se observa la distribución de ambos elementos morfosintácticos respecto a los sujetos, se induce de forma clara que la correlación 
entre L.M.E. y riqueza gramatical no cambiaría. A este respecto, y como comprobación, se han hallado los coeficientes de correlación entre ambos parámetros uniendo en un solo apartado los adjetivos y pronombres demostrativos y en otro apartado únido las dos formas de posesivos, omitiendo las frases negativas, la conjunción que (subordinadas sustantivas) y el relativo que $(r=0,8506)$ $y$, de otra forma, omitiendo los adverbios relativos $(r=0,8399)$.

Riqueza gramatical $\quad \overline{\mathrm{X}}=23,38 \quad \sigma=10,27$

\section{CONCLUSIONES}

(Propuesta para una logopedia de la morfosintaxis)

Es ciertamente presumible que las realizaciones verbales del niño de 2 1/2 años son mucho más que las expresadas en media hora de grabación, aunque se haya intentado, por medio de una serie de materiales y estímulos verbales específicos, hacer que afloren una buena parte de los signos morfosintácticos del castellano.

Por ello, este trabajo está muy lejos de cualquier asomo de finitud respecto a este campo del lenguaje.

A tenor de los resultados obtenidos, pueden presentarse tres conclusiones:

A) Ya que el trabajo se ha hecho fundamentalmente sobre una base fenomenológica y en el capítulo de Resultados se han expuesto los signos morfosintácticos y el porcentaje de niños que los utilizaron, resulta poco interesante recapitular dichos resultados.

Sin embargo, puede ser más práctico considerar cuál es la competencia morfosintáctica presente en la mayoría de los niños de $2 \frac{1}{2}$ años.

- En un $75 \%$ o más, estos niños emplean interrogaciones basadas en la entonación e iniciadas con partículas interrogativas (sobre todo, el pronombre qué).

- Emiten frases negativas.

- Los modelos oracionales más frecuentemente utilizados son $S V$, SVPr, SVCd y SVCa. El Ca es un adverbio de lugar (ahí y aquí). Cuando no se emplean éstos, sino un sustantivo, éste es también un Ca de lugar, conectado dentro del sintagma verbal con la preposición en. La menor presencia de otros tipos de $\mathrm{Ca}$ se debe probablemente a la menor cantidad de información que aportan, es decir a su presunta redundancia: la especificación del tiempo estaría ya presente en el morfema verbal, y el modo y la intensidad en la propia actividad del niño y en los elementos comunicativos no lingüísticos. En cualquier caso, es evidente que la primera especificación circunstancial se refiere al lugar. (Véase, a este respecto, el relativamente alto porcentaje de niños que utilizan la preposición a (dirección).

- Los tiempos del verbo a tener en cuenta en esta descripción tipo son, además de la forma atencional imperativa, el presente de indicativo y pretérito perfecto de indicativo, que, abundando en lo comentado más arriba sobre los $\mathrm{Ca}$ de tiempo, pueden relacionarse con los adverbios de tiempo más frecuentes: abora y ya.

- No se dan, dentro del límite porcentual señalado, oraciones subordinadas, ni coordinadas, a pesar del frecuente empleo de la conjunción y, que cumple la función de nexo en sintagmas nominales heterogéneos y una función fática, podría decirse, ligando las emisiones dentro de un contexto comunicativo.

- Los artículos, con sendas funciones presentadoras del sustantivo, indeter- 
minada y determinada, no plantean especiales dificultades a los niños de 2 1/2 años.

- Referido a éste y a otros signos morfosintácticos es digno de notarse el hecho de que cuando al niño de 3 ó más años se le presentan ítems para evaluar diversos aspectos de la competencia morfosintáctica, por ejemplo, el artículo, no es infrecuente encontrarnos con fracasos, en principio sorprendentes. Esto ha ocurrido en la adaptación del Northwestern Syntax Screening Test (NSST) de L. Lee al castellano (en preparación), en niños de 3 a 7 años. En 1973 R. Brown ya apuntó: aLas evaluaciones de formas concretas de competencia lingüistica basadas en resultados experimentales afechan» las competencias en cuestión más tarde que las evaluaciones basadas en datos de observación directa como la realizada en este trabajo.

- Respecto a los pronombres y adjetivos presentadores, las diferencias en su utilización son poco nítidas y además debe tenerse en cuenta lo dicho sobre el uso de éste,-a + gesto y este, -a y la dificultad de separarlas en dos entidades distintas.

- Se emplean los pronombres personales con función de complemento en posición proclítica y enclítica.

- Probablemente el frecuente empleo del adjetivo otro,-a esté en relación con la función de introducir en un contexto comunicativo conocido una información nueva, ya que el niño tendería a marcar explícitamente ésta y no lo conocido, obvio, o acabado de mencionar (Weisenburger, 1976, citado en Hernández-Pina, 1984).

Las concordancias, relativas al género, número y persona en los verbos, están generalmente bien realizadas.

B) Teniendo en cuenta la hipótesis plausible de que el orden en la adquisición de signos morfosintácticos es consistente interindividualmente (Brown, Johnston y Shery, 1976, citado en Bowerman, 1986), pueden ordenarse nuestros sujetos de menor a mayor riqueza gramatical y pretender que el progresivo enriquecimiento respecto a los signos morfosintácticos observados en este trabajo, es el que se produce en un niño, individualmente considerado. Es decir, si se da un orden, igual para la gran mayoría de los niños, en el desarrollo de la morfosintaxis, es lógico pensar que nuestro $S 1$ (el de menor riqueza gramatical), adquirirá primero los signos morfosintácticos presentes en las emisiones del S2, después los del S2, después los del S3 y así sucesivamente.

Así, se puede proponer un orden de adquisición, teniendo como criterio el porcentaje de niños que emplean cada uno de los signos estudiados:

Interrogación basada en la entonación, no, estructura oracional $S V$, morfema verbal del presente de indicativo, sí, estructura $S V P r, S V C d, S V C a$, éste,-a, etc.

C) Basándonos en este orden, y respondiendo al segundo objetivo del trabajo, se propone un guión, dividido en 10 pasos, que ayude a los logopedas en su actividad con niños que presentan alteraciones en este área del lenguaje.

Por las características de este estudio, parece lógico que este guión será de mayor utilidad en el trabajo con niños afectados de retraso de lenguaje, aunque no existen razones aparentes para no tenerlo en cuenta en los trastornos de lenguaje, considerando con Miller, que éstos trastornos, «pautas lingüisticas que se separan de la secuencia evolutiva habitual y varían respecto a las expectativas que se tienen con respecto a una determinada etapa, quizá no existan en los niños» ya que ael análisis minucioso de la desviación en la conducta lingüística de los niños que padecen esta deviación ha revelado la existencia de pautas evo- 
lutivas semejantes a las de los niños normales, con diferencias fundamentales en el ritmo de adquisición y con una variedad más limitada de las estructuras utilizadas, aunque se consiguieran los principales logros esperables dentro de una etapa lingüísticas.

He aquí la especificación de los diez pasos:

1. Interrogaciones por medio de la entonación

Estructuras de frase: $S V$ y $S P r V$

Verbos: formas activa y reflexiva

tiempos: presente de indicativo

Adverbios: si no.

2.--Estructura de frase: $S V C d S V C a$ (el Ca normalmente es adverbio)

Artículos (predominio casi exclusivo del singular)

Pronombres: demostrativos: éste,-a

Tiempos verbales: pretérito perfecto de indicativo

Adverbios: $a b i$

3. Interrogativas con iniciador (predomina el pronombre que).

Oraciones negativas

Adjetivos presentadores: otro

Pronombres personales en función de complemento

demostrativos: esto

Adverbios: aquí también

Preposiciones: en

Conjunciones: $y$

4. Estructura de frase: SVCdCa

Adjetivos presentadores: demostrativo: este,-a

Pronombres: personales con función de sujeto

demostrativos: ése,-a

indefinido: otro,-a

Forma verbal: gerundio (como $\mathrm{Ca}$, o como parte de un verbo con función "continuativa»)

Adverbios: alli,-a

5. Pronombre: demostrativo: eso

Adverbios: asi

Preposiciones: con, a (dirección), a (con Ci o Cd), de (en locuciones preposicionales o como partitiva), para.

6. Adjetivos presentadores: posesivos

Formas verbales ir a + infinitivo (valor de futuro)

Posesivos como atributo (tras el verbo)

Adverbios debajo, muy (mucho), abora, arriba

Pronombre relativo: que (se inician oraciones subordinadas adjetivas).

7. Estructura de frase: $S V C i$

Adjetivos presentadores: ese,-a

Adverbios: nada, más, encima

Preposiciones: de (materia), de pertenencia) por

Conjunciones: pero

8. Estructuras de frase: $V i C d, V i C d C a, s V C d C i$

Adjetivos presentadores: $m u c b o,-s$

Pronombres: indefinidos: mucho,-s, todo

Tiempos verbales: imperfecto de indicativo, presente de subjuntivo y pretérito indefinido 
Adverbios: ya, entonces, detrás, lejos, adentro y el relativo: donde

Conjunciones: como (comparativa)

9. Estructuras de frase: $V i C a, S V C a C a, S V C d C i C a$

Adjetivos presentadores: indefinidos: poco

Pronombres: indefinido: poco

Tiempos verbales: futuro simple de indicativo

Adverbios: mañana, luego, afuera, bien, tampoco y el relativo: cuando

Preposiciones: de (procedencia) sin

Conjunciones: que, porque

10. Estructuras de frase: SVciCa, SVCaCaCa, SVPrCa... ...

Adjetivos presentadores: indefinidos: algunos,-s, ninguno

Tiempos verbales: potencial simple

Adverbios: siempre, pronto, de noche, tarde, aún, hoy, ayer, mal

Preposiciones: hasta (límite espacial)

Conjunciones: si, para que.

\section{Notas}

1 A veces estas llamadas de atención tienen la forma de imperativo: ¡mira!, pero no es considerada como verbo, núcleo de un predicado con otros elementos elípticos. Sin embargo, en otras ocasiones sí es tomada como tal. Veamos estos dos ejemplos:

S18: emira qué hacen!

*mira qué se ha puesto

S19: «imira!, ¡qué gatos más pequeños!»

El S18 emite dos oraciones compuestas con dos núcleos en forma de imperativo («mira») seguidos de dos complementos directos («que hacen* y «que se ha puestos). El S19, en cambio, emite una llamada de atención (*imira!») y, después de una breve pausa, emite un nombre con modificaciones admirativos, pero que es independiente de la primera exclamación.

\section{Referencias}

Bowerman, M. (1986). Desarrollo semántico y sintáctico. En Schiefelbusch, R. L. (Ed.). Bases of language Intervention. University Park Press, Baltimore (Trad. cast.: Bases de la intervención en el lenguaje. Madrid: Alhambra, 1986).

BRUNER, J. (1983). Child's talk. Learning to use Language, Nueva York y Londres, W.W. Norton. (Trac. cast.: El habla del niño, Barcelona: Paidós, 1986).

CAZDEN, C. B. y BROWN, R. (1975). El desarrollo temprano de la lengua materna. En Ienneberg, E.H. y Lenneberg, E.H. y Lenneberg, E, (eds.): Foundations of Language Development-A multidisciplinary Approach I, II. Unesco, (Trad. cast.: Fundamentos del desarrollo del lenguaje, Madrid: Alianza, 1982).

CRYSTAL, D. FletCHER, P. y GARMAN, M. (1981). The Grammatical analysis of Language disability, Vol. I de la serie Studies in language disability and remediation. Londres: Edward Arnold. (Trad. cast.: Análisis gramatical de los trastomos del lenguaje, Barcelona, Ed. Médica y Técnica, 1983).

GIL GAYA, S. (1974). Estudios de lenguaje infantil, Barcelona: Bibliograf.

Hernandez PINA, F. (1984). Teorías psicosociolingüísticas y su aplicación a la adquisición del español como lengua materna. Madrid: Siglo XXI de España.

HeRnandez Pina, F. (1984). Sobre la universalidad de las categorías eje-abierta (pivot-open). En Siguan, M (ed.): Estudios sobre Psicología del lenguaje infantil, Madrid: Pirámide.

LAmIQUiz, V. (1975). Lingü̈́stica Española, Sevilla: Publicaciones de la Universidad de Sevilla.

LEE, L. (1971). Northwestern Syntax Screening Test. Evanston: Northwestern University.

LOPEz ORNat, S. (1984). La expresión de límites. En Siguan M.: (ed) Estudios sobre Psicología del lenguaje infantil, Madrid: Pirámide.

MILLE, J. F. Evaluación de la conducta lingüística en los niños. En Schiefelbusch, R.L. (Ed.) Bases of Language Intervention. Baltimore. University Park Press. (Trad. cast.: Bases de la intervención en el lenguaje. Madrid: Alhambra, 1986).

Richelle, M. (1971). L'acquisition du langage. Bruselas, Dessart. (Trad. cast.: La adquisición del lenguaje. Barcelona: Herder, 1975).

SOLER, M. R. (1984). Adquisición y utilización del artículo. En Siguan, M. (ed.): Estudios sobre Psicología del lenguaje infantil, Madrid: Pirámide. 


\section{Extended summary}

This study is part of a wider research project on the development of morphosyntax in children between $21 / 2$ and 7 years old.

The study, is based on an adaptation of the Northwestern Syntax Screening Test (NSST) by Laura Lee for children from 3 years onwards, to this items to explore morphosyntactic signs missing from the English language but present in Spanish have been added, as well as other items which include other morphological and syntactic aspects considered to be important.

As this test was not ideal for evaluating $2 \frac{1}{1 / 2}$ years olds, another approach was adopted to provide an individualised character to the study.

The aim of the study is two-fold: First, to explore the morphosyntactic competence of $21 / 2$ year old children, which is considered to be a key age because of syntactic expansion, and to find out if the Mean Length of Utterance (M.L.U.) might be a good measure of the morphosyntactic complexity in the speaking infant. Secondly to provide an instrument for speech therapy related to later or faulty acquisition of grammar.

The intention was not to make an exhaustive analysis of syntax, the study covered what could be considered as the formal structure, independent of the meaning of the utterances.

The technique used was a recordinga of a half-hour conversation between the tester and each of the children. There were 24 children, both boys and girls all of whom were $21 / 2$ years old \pm 7 , days. All attended state kindergartens in Pamplona.

Two-thirds of each conversation was semi-directed using the same material for all the children: a story, the graphic NSST material and a few toys associated with the story line of the story (activities and characters on a farm during a 12 month span). This material and guidance were necesary to distinguish all the morphosyntatic elements to be studied.

These elements can be grouped into 13 categories:

1. Interrogative. Questions based only on intonation is present in all the children. Nevertheless, the number of children who begin questions with interrogative particles is lower $(79,17 \%)$. These results are logical taking into account the supra-segmentary phenomenon of the interrogative pitch in the stuttering stage.

2. Negative. All the children used the adverb NO. The use of complete negative statements was lower $(79,17 \%)$. The use of not... either is far less frequent than the affirmative yes..., toolalso. The use of neither is non existent.

3. Sentence Models. The most frequent (100\%) is the Subject-Verb model the Subject-verb-predicate using a linking verb like to be is used in 23 of the 24 children.

The Subject verb-direct object and Subject-verb-adverb object are used $91,66 \%$ of the time. The adverbial object is usually an adverb of place, time or manner.

The Subject verb-direct object-adverbial object is used by $62,5 \%$.

Other sentence models are quite low (less than $30 \%$ ). For example Subjectverb-indirect object direct-object, etc.

Passive sentences were neither used nor understood by any of the children.

4. Coordination. For each child the number of coordinates was calculated 
( = number of compound coordinating sentencesitotal number of sentences $\times 100) ; 41,66 \%$ of the children used compound structures. At this age the juxtaposition has a coordinate as well as subordiante value in many cases, although this was not considered in the study.

5. Subordination. Using the same formula as in 4 above, the subordination index was derived. They were analyzed one by one. The most frequent kind was relative clauses. Some «because clauses and «strong» ellipsis were also found.

6. Gender and number. Generally these tend to be used correctly.

7. Articles, Except with subjects where they are non-existent, and in two other cases where the aprotoarticles $(/ \mathrm{a} /)$ is used, the rest of the children used articles correctly, especially in the singular.

8. Demonstrative Adjectives. The demonstrative this (the most frequent); that was non-existent, were checked, as well as the possessive adjectives and the indefinite and numeral quantifiers.

9. Pronouns. The percentage of subject pronouns and personal direct object pronouns in proclitic and enclitic position was found. With the demonstrative pronouns there existed the doubt as to the children's capacity to distinguish them but one must consider that at this age, gesture means a great deal regarding the object being referred to.

The indefinite pronouns where also studied.

10. Verbs. Tense and mood were observed. The most frequent tenses used by a child of $21 / 2$ years are the present simple, present continuous, the simple past and present perfect. The future, is expressed with a periphrasis based on the present (go + infinitive of verb). Other tense forms are much less frequent.

11. Adverbs. The adverbs studied were those indicating negation, affirmatives, place, time, manner, intensity, relatives and interrogatives. In children of this age $(21 / 2)$, except adverbs of place, which appear to have a quite high frequency of use, the other adverbs are usually only used in one form.

12. Prepositions. The different prepositional words as well as the varied meaning of each were studied.

13. Conjunctions. Very closely related to sections 4 and 5 above, all the conjunctions recorded on the tape were counted and studied.

In addition, concerning the second aspect of the primary objective, the coefficiente of correlation between the grammatical richness (understood as the sum of morphosyntactic elements found in each child) and his/her M.L.U.

$\overline{\mathrm{X}}=2,33, \mathrm{DS}=0,78$ ) the result was $\mathrm{r}=0,8402, \mathrm{p} 0,01$, which makes the M.L.U. a valid measure of grammatical richness. Besides, this coefficient sustains the concept of grammatical complexity in an saccumulative sense» or a product of the necessity to limit or confine the meaning/message to an utterance, adding words, using subordinating constructions or comparisons, etc. to get the message across.

Regarding the second objective, keeping in mind the hypothesis that the order of the adquisition of morphosyntactic signs is consistent amongst individuals, our studies can gauge the children's ability from least to best in grammatical competence and venture that progressive morphosyntactical richness observed in this study is what is produced in a child, when considered individually.

Therefore, based on this order, a guide is proposed which is broken down into 10 steps or phases. In these, which morphosyntactic signs should be considered are specified. It is hoped that this breakdown will help speech teraphists in their work with children who have deficiencies in this area of language. 